\title{
Small airways and early origins of COPD: pathobiological and epidemiological considerations
}

\author{
Francesca Polverino ${ }^{1}$ and Joan B. Soriano $\mathbb{1}^{2,3}$ \\ Affiliations: ${ }^{1}$ Asthma and Airway Disease Research Center, Dept of Medicine, University of Arizona, Tucson, \\ AZ, USA. ${ }^{2}$ Hospital Universitario de la Princesa, Universidad Autónoma de Madrid, Madrid, Spain. ${ }^{3}$ Centro de \\ Investigación en Red de Enfermedades Respiratorias (CIBERES), Instituto de Salud Carlos III (ISCIII), Madrid, \\ Spain.
}

Correspondence: Francesca Polverino, Asthma and Airway Disease Research Center, Dept of Medicine, University of Arizona, Tucson, AZ, USA. E-mail: fpolverinolacopdnet.org

@ERSpublications

The role of the small airways and the early origins of airflow limitation http://bit.ly/35Uaib3

Cite this article as: Polverino F, Soriano JB. Small airways and early origins of COPD: pathobiological and epidemiological considerations. Eur Respir J 2020; 55: 1902457 [https://doi.org/10.1183/13993003.024572019].

Until recently, the conceptual model of the natural history of COPD has been greatly focused on an accelerated decline of lung function, which would occur in adult life in susceptible individuals when chronically exposed to environmental noxious agents, such as cigarette smoke and other inhaled gases. However, $25 \%-45 \%$ of all COPD patients worldwide have never smoked [1]. In addition, pre- and peri-natal factors associated with diverse biological mechanisms can determine low lung function in young adults [2-4]. Among these factors, asthma and active and/or passive smoking act synergistically to affect early lung function deficits in young adulthood $[5,6]$. The impact of smoke exposure in susceptible smokers is detectable with exposures to as little as 8-10 pack-years, in their late $30 \mathrm{~s}$ to early $40 \mathrm{~s}$ [7]. Similarly, early onset asthma is observed in $26 \%$ of those diagnosed with COPD at mean age of 37 years, which represents a 20 -fold increased risk of adult airway obstruction compared with those without asthma diagnosis [8]. However, what the combined effects of current active smoking and asthma are in a population of young adults is unknown.

The paper by ARSHAD et al. [9] in this issue of the European Respiratory Journal addresses this knowledge gap. The authors examined the Isle of Wright Birth Cohort at ages 10, 18 and 26 years for lung function and asthma, while information on environmental exposures, including active and passive smoking, were obtained since birth. The presence of current asthma and/or current smoking at age 26 years was used as rationale to divide the population into four groups (non-smokers without asthma; non-smokers with asthma; smokers without asthma; and smokers with asthma), independently of their previous history of smoking or asthma; further, they retrospectively assessed their previous lung function in order to assess their risk of future COPD. These investigators should be praised for following up children from birth, with extensive questionnaires, both pre- and post-bronchodilator spirometry, and with a decent retention ( $>70 \%$ at age 26 years, as per their table S2), all following STROBE guidance for observational research. And, last but not least, for successfully running a cohort for a quarter of a century, and counting [10]. Indeed, several limitations are inherent, such as the recall bias for previous smoke exposure in a population which is classified solely according to their current smoking status. Nonetheless, this study 
delivered important outcomes. Predictably, subjects with both asthma and smoking at the age of 26 years had the poorest lung function, but had already low lung function very early in life (age of 10 years). This suggests that asthma in early adulthood is preceded by changes in lung function which can be tracked back to many years previously, and which need to be identified early, as they might lead to future early development and (mis)diagnosis of COPD.

A novel aspect of this study is the longitudinal assessment of the mean forced expiratory flow between $25 \%$ and $75 \%$ of the forced vital capacity $\left(\mathrm{FEF}_{25-75}\right)$, an indicator of small airway disease which is affected early in smoking-related lung disease. For years, the $\mathrm{FEF}_{25-75}$ has been underused in clinical cohort studies due to its high rate of variation among subjects. However, others have shown that the $\mathrm{FEF}_{25-75}$ is highly sensitive to early lung changes leading to airflow limitation [5]. In the current study, $\mathrm{FEF}_{25-75}$ and forced expiratory volume in the first second $\left(\mathrm{FEV}_{1}\right)$ /forced vital capacity $(\mathrm{FVC})$ ratio were more sensitive than $\mathrm{FEV}_{1}$ alone in detecting early lung function impairment in young adult smokers. This aspect calls for an important consideration: the decline in indices of lung function reflecting airway diameter occurs early in adult life and should be evaluated more frequently within the assessment of lung volumes in cohort studies of young adults at risk for early COPD.

The early changes in $\mathrm{FEF}_{25-75}$ and $\mathrm{FEV}_{1} / \mathrm{FVC}$ mirror the contribution of the small airways in the pathogenesis of early lung function limitation, and might therefore be a link between childhood asthma and early COPD. Regrettably, many knowledge gaps in the pathogenesis of early airflow limitation still exist, mainly because COPD has always been considered a disease of the elderly, and little attention has been paid to the pathological changes occurring in the lungs of individuals at risk before they develop clinically evident COPD [2]. COPD is still considered as a clinical disorder associated with large airway disease (chronic bronchitis) and alveolar loss (emphysema). However, it later became apparent that the small conducting airways of less than $2 \mathrm{~mm}$ in internal diameter (terminal bronchioles) are the major site of airflow obstruction in susceptible smokers, leading to accelerated loss of lung function in the early stages of COPD (figure 1) [11]. Given the intrinsic limitations of obtaining biopsies, the introduction of micro computed tomography (micro-CT) has made it possible to show that a reduction in both terminal and transitional bronchioles is well established before emphysematous destruction is evident (figure 2) [12], and even before the subjects meet the spirometric criteria for COPD. Interestingly, small airway remodelling may also be a primary event in asthma pathogenesis if it is the result of disturbed lung development. The small airway wall structure can be altered very early in life, and can indeed precede airway wall inflammation and thus may present as a primary event in asthmatic lungs [13, 14]. Understanding how early life, or even prenatal, risk factor exposures may be mechanistically linked to

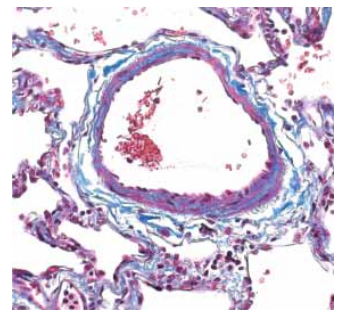

Normal small airways

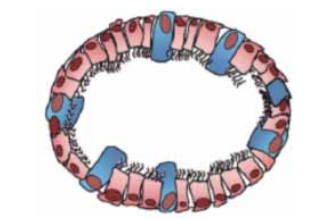

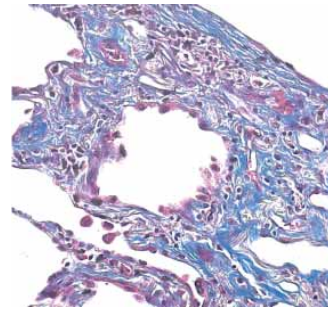

Remodelling and loss of small airways

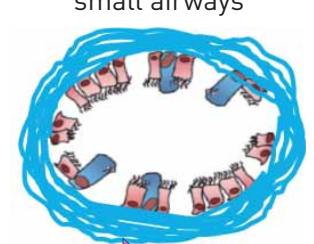

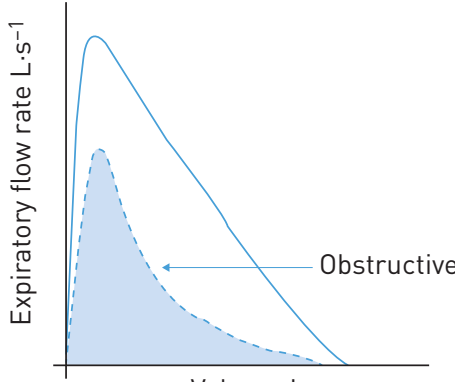

Volume L

\begin{tabular}{|c|c|c|}
\hline & $\downarrow \mathrm{FEV}_{25-75}$ & $\downarrow \mathrm{FEV}_{1}$ \\
\hline Normal & Early COPD & Late COPD
\end{tabular}

FIGURE 1 Pathology samples of respiratory biopsies. The small airways are the major site of airflow obstruction in susceptible smokers, leading to accelerated loss of lung function in the early stages of COPD. Representative pictures of a) a small airway in a 43-year-old never smoker (female); and b) a small airway with severe remodelling in a 45-year-old smoker without COPD (male). Trichrome staining of formalin-fixed paraffine embedded sections. Magnification 40x. The blue staining represents areas of remodelling around the airways. $\mathrm{FEF}_{25-75}$ : forced expiratory flow between $25 \%$ and $75 \%$ of forced vital capacity; $\mathrm{FEV}_{1}$ : forced expiratory volume in the first second. 


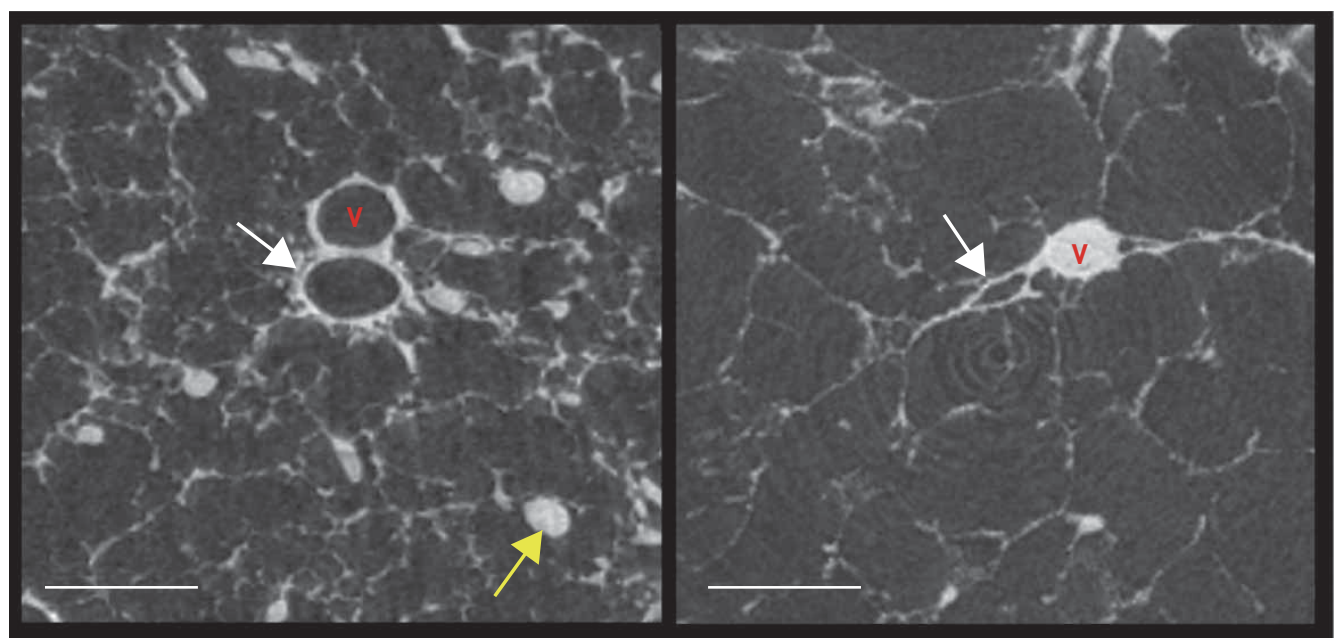

FIGURE 2 Micro computed tomography (micro-CT) scans of lungs. Representative cross-sectional images from age and sex matched subjects (control: 52-year-old female; COPD: 47-year-old female). Scale bar $1 \mathrm{~mm}$. White arrows indicate terminal bronchiole; v: blood vessel (in controls, blood is flushed out); yellow arrow: ice crystal. Credit to Dragoș M. Vasilescu and Tillie-Louise Hackett from the Centre for Heart Lung Innovation, The University of British Columbia, Canada.

small airway structural and functional changes later in life will be both highly interesting from a scientific perspective as well as highly important for understanding how bronchial asthma evolves into fixed airflow limitation and early COPD.

Future clinical cohorts and therapeutic trials might target younger subjects with low lung function before they meet the spirometric criteria for COPD [3], as trials on older, more severe COPD may have failed because they were initiated in patients with COPD who already had "irreversible disease", characterised by established small airway remodelling. A clear understanding of the cause of early airflow limitation leading to COPD could lead to more personalised strategies for subclinical disease [15], and preventive therapies that ameliorate the underlying pathological process rather than the symptoms alone.

Support statement: This work was supported by the Flight Attendant Medical Research Institute (grant: YFAC14004), by the Parker B. Francis Foundation, and by the Asthma and Airway Disease Research Center at University of Arizona. Funding information for this article has been deposited with the Crossref Funder Registry.

Conflict of interest: None declared.

\section{References}

1 Lamprecht B, McBurnie MA, Vollmer WM, et al. COPD in never smokers: results from the population-based burden of obstructive lung disease study. Chest 2011; 139: 752-763.

2 Soriano JB, Polverino F, Cosio BG. What is early COPD and why is it important? Eur Respir J 2018; 52: 1801448

3 Lange P, Celli B, Agusti A, et al. Lung-function trajectories leading to chronic obstructive pulmonary disease. N Engl J Med 2015; 373: 111-122.

4 Bui DS, Lodge CJ, Burgess JA, et al. Childhood predictors of lung function trajectories and future COPD risk: a prospective cohort study from the first to the sixth decade of life. Lancet Respir Med 2018; 6: 535-544.

5 Guerra S, Stern DA, Zhou M, et al. Combined effects of parental and active smoking on early lung function deficits: a prospective study from birth to age 26 years. Thorax 2013; 68: 1021-1028.

6 Voraphani N, Stern DA, Wright AL, et al. Risk of current asthma among adult smokers with respiratory syncytial virus illnesses in early life. Am J Respir Crit Care Med 2014; 190: 392-398.

7 GBD 2015 Chronic Respiratory Disease Collaborators. Global, regional, and national deaths, prevalence, disability-adjusted life years, and years lived with disability for chronic obstructive pulmonary disease and asthma, 1990-2015: a systematic analysis for the Global Burden of Disease Study 2015. Lancet Respir Med 2017; 5: 691-706.

8 Aanerud M, Carsin AE, Sunyer J, et al. Interaction between asthma and smoking increases the risk of adult airway obstruction. Eur Respir J 2015; 45: 635-643.

9 Arshad SH, Hodgekiss C, Holloway JW. Association of asthma and smoking with lung function impairment in adolescence and early adulthood: the Isle of Wight Birth Cohort Study. Eur Respir J 2020; 55: 1900477.

10 Kohansal R, Soriano JB, Agusti A. Investigating the natural history of lung function: facts, pitfalls, and opportunities. Chest 2009; 135: 1330-1341.

11 McDonough JE, Yuan R, Suzuki M, et al. Small-airway obstruction and emphysema in chronic obstructive pulmonary disease. $N$ Engl J Med 2011; 365: 1567-1575. 
12 Koo HK, Vasilescu DM, Booth S, et al. Small airways disease in mild and moderate chronic obstructive pulmonary disease: a cross-sectional study. Lancet Respir Med 2018; 6: 591-602.

13 Fehrenbach H, Wagner C, Wegmann M. Airway remodeling in asthma: what really matters. Cell Tissue Res 2017; 367: 551-569.

14 Carr TF, Altisheh R, Zitt M. Small airways disease and severe asthma. World Allergy Organ J 2017; 10: 20.

15 Polverino F, Hysinger EB, Gupta N, et al. Lung MRI as a potential complementary diagnostic tool for early COPD. American J Med 2020; in press [https:/doi.org/10.1016/j.amjmed.2019.12.009]. 